\title{
Determinación de algunas propiedades físicas y mecánicas de Grevillea robusta A. Cunn. del estado de Veracruz
}

\author{
Reyna Paula Zárate Morales ${ }^{1}$ \\ Víctor Rubén Ordóñez Candelaria ${ }^{1}$ \\ José Luis Martínez Castillo ${ }^{1}$
}

\begin{abstract}
RESUMEN
Se determinaron las propiedades físicas y mecánicas de la especie Grevillea robusta A. Cunn. con especímenes colectados en la región central del estado de Veracruz. Las propiedades mecánicas se estimaron en condición "verde" y seca. Se determinó que hay un valor alto de la relación entre la contracción tangencial y la radial, lo que generalmente se interpreta como una baja estabilidad dimensional; sin embargo, del secado del material para pruebas mecánicas en condición seca, se obtuvieron piezas con distorsiones pequeñas. Las propiedades mecánicas que se estudiaron fueron flexión estática, compresión paralela y perpendicular a la fibra, dureza, cortante paralelo a la fibra y tenacidad por impacto. Los resultados que se presentan son los valores promedio de cada propiedad, el número de ensayos realizados para obtenerlos, la desviación estándar y el coeficiente de variación, tanto en condición seca como en condición "verde". Considerando la presencia abundante de radios multiseriados apreciables a simple vista, se estimó el porcentaje del área de una sección tangencial ocupada por dichos radios, encontrando valores altos de este porcentaje que, posiblemente, influyen en la resistencia mecánica de la madera de esta especie. Se encontró que esta madera tiene una resistencia mecánica de baja a media.
\end{abstract}

PALABRAS CLAVE:

Grevillea robusta, propiedades físicas y mecánicas, pequeñas probetas, contracciones, Veracruz.

\begin{abstract}
The physical and mechanical properties of Grevillea robusta A. Cunn. were determined, testing small clear specimens. The testing material was obtained from two trees collected in the central region of Veracruz state. The mechanical properties were determined in green and dry condition. The material for tests in dry condition was obtained from the drying tests. A high ratio between tangential and radial contraction was calculated, which could be construed as an indication of low dimensional stability; however, from these experiments pieces with minimum distortions resulted. The tests carried out were: static bending, compression and shear parallel to grain, compression perpendicular to grain, end hardness and side hardness (radial and tangential directions) and toughness. In this paper the mean values, the standard deviation and the coefficients of variation are reported for all properties mentioned. Taking into account the abundance of multiseriate rays which can be with the naked eye, and estimate of the percentage of area in the tangential section was determined and high values of this percentage were found which might have an influence on the mechanical strength of the of this species. It was found that this wood has a strength from low to medium.
\end{abstract}

KEYWORDS:

Grevillea robusta, physical and mechanical properties, small clear specimens, shrinkage, Veracruz. 


\section{INTRODUCCIÓN}

Grevillea robusta es una especie nativa del este de Australia, que se utiliza extensivamente como sombra en plantaciones de café o té y como especie ornamental en regiones tropicales y subtropicales (Chudnoff, 1984). Los ejemplares cultivados de esta especie pueden alcanzar alturas hasta de $12 \mathrm{~m}$ (Nee, 1988) con ramificaciones a baja altura. En la figura 1 se presenta un ejemplar de la especie en pie. En el estado de Veracruz se cultiva principalmente en zonas cercanas a la ciudad de Xalapa; en la figura 2 se presenta un croquis de la distribución de esta especie en el Estado. En Veracruz se le conoce comúnmente como grevilea, grevilia 0 gravilea y se utiliza frecuentemente para proporcionar sombra en plantaciones de café y para fines ornamentales (Niembro, 1986). Su madera tiene un color pardo rosáceo que cambia a amarillo parduzco con la exposición y a simple vista se puede apreciar que tiene gran cantidad de radios.

\section{OBJETIVO}

Determinar las propiedades mecánicas de la madera de Grevillea robusta A. Cunn. de la región central del estado de Veracruz, así como algunas de sus propiedades físicas, con el fin de proponer sus posibles usos.

\section{METODOLOGÍA}

Colecta. Se colectaron dos árboles de 6 y $7 \mathrm{~m}$ de fuste potencialmente comercial, respectivamente, ambos con $35 \mathrm{~cm}$ de diámetro a la altura del pecho (DAP). De acuerdo con Ordóñez et al. (1989), con este tamaño de la muestra se obtiene una probabilidad del $90 \%$ y límites de confianza de $28 \%$ para coeficientes de

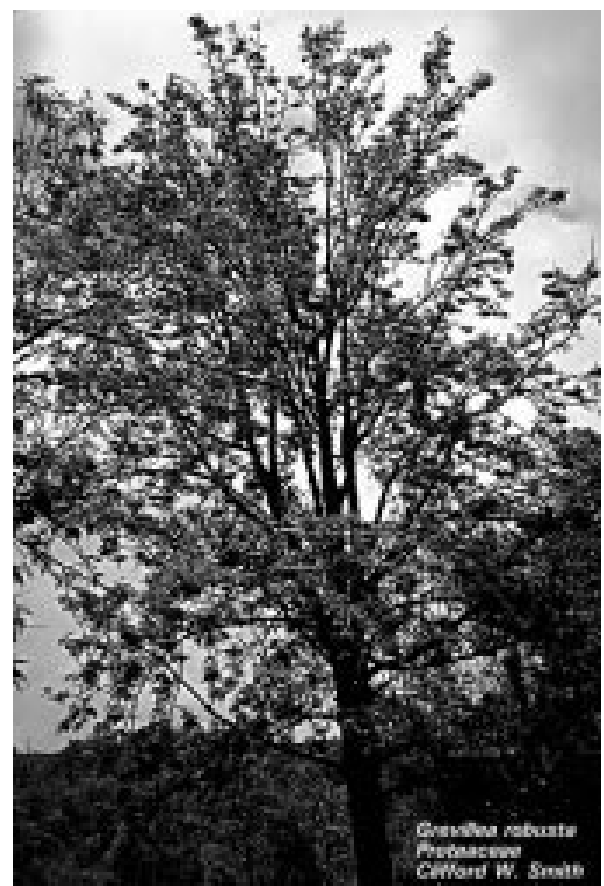

Figura 1. Grevillea robusta A. Cunn. en pie.

variación de $10 \%$, en la estimación de sus propiedades físicas y mecánicas. La colecta se realizó en una plantación de café en las afueras de la ciudad de Xalapa, en una superficie sensiblemente horizontal de suelo franco con materia orgánica abundante color café oscuro, a una altitud de 1350 msnm. Cada fuste se cortó siguiendo el patrón de corte que se muestra en la figura 3 y las piezas se marcaron con las letras que se indica en la misma figura. Este patrón de corte concuerda con el propuesto en el manual de procedimientos de prueba de este laboratorio (INECOL, 2000), con la excepción de que en lugar de obtener una cuarta troza para pruebas mecánicas, se obtuvo una tercera pieza para pruebas de química y anatomía. Esto se debió al corto fuste comercial de los árboles. 




Figura 2. Mapa de distribución de Grevillea robusta A. Cunn. en el estado de Veracruz. Tomada de Nee (1988).

Las piezas A, B y C (trozas) se utilizaron para las pruebas mecánicas, de las piezas A', B' y C' (rolletes) se obtuvieron las probetas para contracciones y se almacenó material para posteriores pruebas de anatomía y química de la especie. Las piezas marcadas con los números 1 y 2 se utilizaron para calcular el contenido de humedad del árbol al momento de la colecta. Todo el material fue trasladado de inmediato a las instalaciones del laboratorio donde se realizó esta investigación. Los extremos de las piezas que se utilizarían para las pruebas mecánicas fueron cubiertos con una capa de pintura de aceite para evitar grietas por secado drástico durante el lapso entre su llegada a las instalaciones del laboratorio y su procesamiento. Los rolletes se mantuvieron sumergidos en agua hasta el momento de cortar las probetas de contracciones.
Maquinado. La evaluación del maquinado, se realizó durante la elaboración de las probetas para los ensayos físicos y las mecánicos. Los defectos que se calificaron en cada probeta en operaciones de cepillado y lijado fueron grano algodonoso, grano arrancado, grano levantado y marcas de viruta, de acuerdo con la clasificación propuesta por Longwood (1961).

Contracciones. De las piezas A', B' y C' se cortaron rodajas de $2.5 \mathrm{~cm}$ de espesor y de ellas se obtuvieron probetas con las dimensiones especificadas por la Norma ASTM D143 (1997) para las pruebas de contracciones radial y tangencial $(2.5$ por 2.5 por $10.0 \mathrm{~cm}$ ). Las caras transversales de las probetas se cubrieron con una capa delgada de parafina para evitar distorsiones excesivas. 


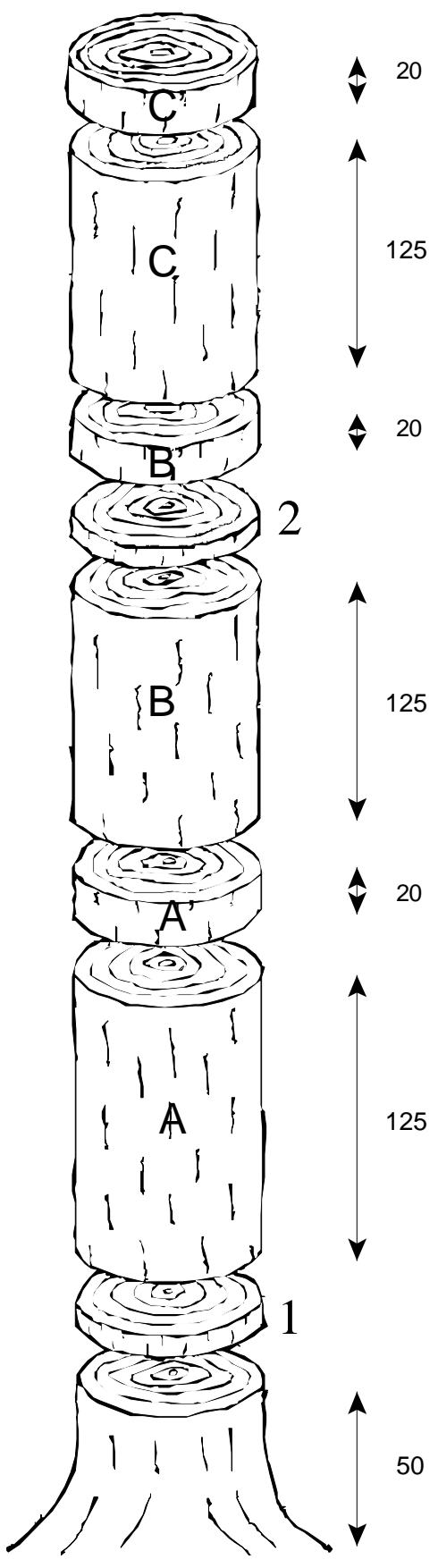

Figura 3. Patrón de corte de los árboles.
Se registraron el peso, el volumen y la longitud iniciales de cada probeta. Se inició el proceso de secado en un ambiente al aire libre con $78 \pm 2 \%$ de humedad relativa $(\mathrm{HR})$ y $19 \pm 1{ }^{\circ} \mathrm{C}$ de temperatura. Del lote completo de probetas para contracciones, se seleccionó al azar un número de piezas, cuyo peso se registró semanalmente hasta obtener un peso constante. Al cabo de cuatro semanas, tiempo en que se logró una variación mínima de peso, se trasladaron todas las probetas a un ambiente seco de $61 \pm 1 \%$ de HR y $20 \pm$ $1{ }^{\circ} \mathrm{C}$ de temperatura, manteniéndose ahí hasta alcanzar peso constante. Las probetas se trasladaron entonces a un ambiente controlado con condiciones estables $\left(45 \%\right.$ de HR y $21 \pm 1{ }^{\circ} \mathrm{C}$ de temperatura). Al alcanzar un peso estable (condición a la que se le designó intermedia, I) se registraron peso y longitud de todas las probetas. Por último, se secaron en un horno de laboratorio con circulación de aire a una temperatura de $103 \pm 3{ }^{\circ} \mathrm{C}$ durante 48 horas, al cabo de las cuales se registraron su peso y longitud finales.

Las contracciones se obtuvieron con las siguientes ecuaciones:

$$
\begin{gathered}
C I=\frac{L_{v}-L_{i}}{L_{v}} \cdot 100 \\
C T=\frac{L_{v}-L_{a}}{L_{v}} \cdot 100
\end{gathered}
$$

donde:

$$
\begin{aligned}
& \mathrm{Cl}= \text { Contracción intermedia (de } \\
& \begin{array}{l}
\text { condición "verde" a condición } \\
\text { intermedia), expresada }
\end{array} \\
& \text { en porcentaje } \\
& \mathrm{CT}= \begin{array}{l}
\text { Contracción total (de condición } \\
\text { "verde" a condición anhidra), } \\
\text { expresada en porcentaje }
\end{array} \\
& \mathrm{L}_{v}= \text { Longitud de la probeta en } \\
& \text { condición "verde" (cm) }
\end{aligned}
$$


condición intermedia $(\mathrm{cm})$

$\mathrm{L}_{\mathrm{a}}=$ Longitud de la probeta en

condición anhidra $(\mathrm{cm})$

Además de los valores de contracción, se obtuvo la densidad relativa ( $\mathrm{pa} / \mathrm{vv})$ de cada espécimen.

A partir de los resultados obtenidos de contracciones radiales y tangenciales, se calculó el punto de saturación de la fibra (PSF). Este se calculó, como lo proponen Choong y Achmadi (1991), promediando el PSF obtenido de las contracciones radiales con el PSF obtenido de las contracciones tangenciales.

Pruebas mecánicas. Elaboración de las probetas. Las piezas $\mathrm{A}$, B y $\mathrm{C}$ se cortaron en tablas de $3.5 \mathrm{~cm}$ y tablones de $6.5 \mathrm{~cm}$ de espesor. Los tablones se cortaron en prismas de sección cuadrada de $6.5 \mathrm{~cm}$ de lado. Del total del material se seleccionó una parte para elaborar probetas a ensayar en condición "verde" y otra para elaborar probetas a ensayar en condición seca. Las piezas se mantuvieron sumergidas en agua durante la elaboración de las probetas en condición "verde" y, posteriormente, las piezas para probetas en condición seca se apilaron para su secado.

Para cada condición se obtuvieron de 2 a 3 probetas por tipo de prueba por troza, excepto para tenacidad por impacto, para la que se obtuvieron en promedio 7 probetas por troza. De los prismas se elaboraron probetas, siguiendo lo establecido en la norma ASTM D143 (1997), para compresión paralela, compresión perpendicular (utilizadas también para dureza) y cortante paralelo. De las tablas de $3.5 \mathrm{~cm}$ se obtuvieron los especimenes para las pruebas de tenacidad por impacto, además de los de flexión estática, los cuales se dimensionaron de acuerdo con los métodos secundarios, debido a los diámetros pequeños de los árboles.
Secado del material a probar en condición seca. Las piezas se dimensionaron a una longitud uniforme cubriéndose en sus extremos con una capa de parafina líquida, para formar una pila que se colocó en un sitio horizontal, al aire libre, protegido de la lluvia y los rayos directos del sol. La pila se orientó perpendicularmente a la dirección de los vientos dominantes de la ciudad de Xalapa. Se ejerció presión sobre la pila mediante tensores que se iban ajustando conforme la madera se iba contrayendo, con el fin de evitar distorsiones excesivas en las piezas. La pila de secado se mantuvo así durante tres meses aproximadamente; al término de este tiempo la madera alcanzó contenidos de humedad de $21 \%$ hasta $27 \%$.

Después del secado al aire libre, el material se pasó a un ambiente controlado con una humedad relativa promedio de $45 \%$ y una temperatura promedio de $25{ }^{\circ} \mathrm{C}$ por espacio de 6 semanas, después de las cuales se elaboraron las probetas.

Realización de pruebas. Las pruebas mecánicas se realizaron en condición "verde" y en condición seca siguiendo lo establecido en el manual de procedimientos de prueba de este laboratorio (INECOL, 2000), basado principalmente en la Norma ASTM D143 (1997). Para la realización de estas pruebas se utilizó una máquina universal de ensayos con 25 t de capacidad, excepto para las de tenacidad por impacto que se realizaron en una máquina de cadena del tipo FPL (ASTM, 1997). El registro de la carga y deformación en las pruebas de flexión estática, compresión perpendicular y cortante paralelo en condición "verde" se hizo mediante el graficador de la máquina universal, el cual genera una gráfica carga-deformación en el momento en que se realiza la prueba y también mediante la carátula de carga de la misma máquina. El mismo registro para las pruebas en condición seca, así como los 
de compresión paralela en condición verde, se obtuvo con un sistema de adquisición de datos (SAD) que graba las señales de los sensores de carga y deformación en un archivo de computadora, al tiempo que genera una gráfica carga-deformación en tiempo real en la pantalla de la computadora. Para los casos de flexión estática, compresión paralela y compresión perpendicular, se obtuvieron de sus respectivas gráficas carga-deformación la carga y la deformación en el límite de proporcionalidad para el cálculo de resultados.

En el caso de las pruebas de tenacidad por impacto, se aplicaron cargas de impacto con un ángulo inicial del péndulo de $60^{\circ}$ para las probetas en condición "verde" y de $45^{\circ}$ para las probetas en condición seca, en ambas condiciones se utilizó la posición del peso del péndulo más desfavorable para la probeta (el máximo brazo de palanca permitido por la máquina) y un claro de carga de $25.4 \mathrm{~cm}$.

Determinación de contenido de humedad $y$ densidad relativa. Al término de cada prueba se obtuvo una porción de cada probeta para determinar el contenido de humedad al momento de la prueba así como la densidad relativa (pa/vv). Las porciones se pesaron inmediatamente después del corte. En el caso de pruebas en condición "verde", de inmediato se registró el volumen verde (vv) de las porciones; en caso de las pruebas en condición seca, las probetas se sumergieron en agua hasta alcanzar el punto de saturación de la fibra y, entonces, se registró su volumen saturado. El peso anhidro de las porciones se obtuvo después de secarlas en una estufa de laboratorio con circulación de aire, a $103 \pm 3{ }^{\circ} \mathrm{C}$ durante 48 horas.

La medición del volumen verde de las porciones se realizó con el método de inmersión.
El contenido de humedad se obtuvo con la siguiente ecuación:

$$
C H=\frac{P I-P A}{P A} \cdot 100
$$

Donde:

$$
\begin{aligned}
\mathrm{CH}= & \begin{array}{l}
\text { Contenido de humedad al } \\
\text { momento de la prueba }(\%)
\end{array} \\
\mathrm{PI}= & \begin{array}{l}
\text { Peso de la porción de probeta al } \\
\text { momento de la prueba }(\mathrm{g})
\end{array} \\
\mathrm{PA}= & \begin{array}{l}
\text { Peso anhidro de la porción de } \\
\text { probeta }(\mathrm{g})
\end{array}
\end{aligned}
$$

Determinación del porcentaje de radios. Hasta este etapa de la investigación no se han realizado estudios de anatomía de la especie, sin embargo, dados los resultados de resistencia mecánica y contracciones y la abundante presencia de radios, se decidió hacer una estimación preliminar del porcentaje de área que ocupan estos radios en una sección. Con un scanner se tomaron imágenes de las secciones tangenciales de 9 piezas de 2.5 por $2.0 \mathrm{~cm}$ aproximadamente, cada una cortada de la parte adyacente a las probetas de flexión estática, obteniendo imágenes a color como la que se presenta en la figura 4a. Se hizo una conversión de ésta a una imagen en blanco y negro, como la que se presenta en la figura $4 b$, para marcar el contraste entre fibras y radios. Utilizando el programa Windows Scanner, que es un analizador de imágenes, se calcularon las áreas en blanco y se calculó su porcentaje con respecto al área total de la sección. En las mismas secciones tangenciales se midió el número de radios por centímetro.

\section{RESULTADOS}

Maquinado del material durante la elaboración de las probetas. La superficie de las probetas obtenidas de operaciones de cepillado y lijado presentó una apariencia con calificación de excelente a buena y con escasa presencia de nudos grandes. Desarrolla en poca proporción el defecto de grano levantado 


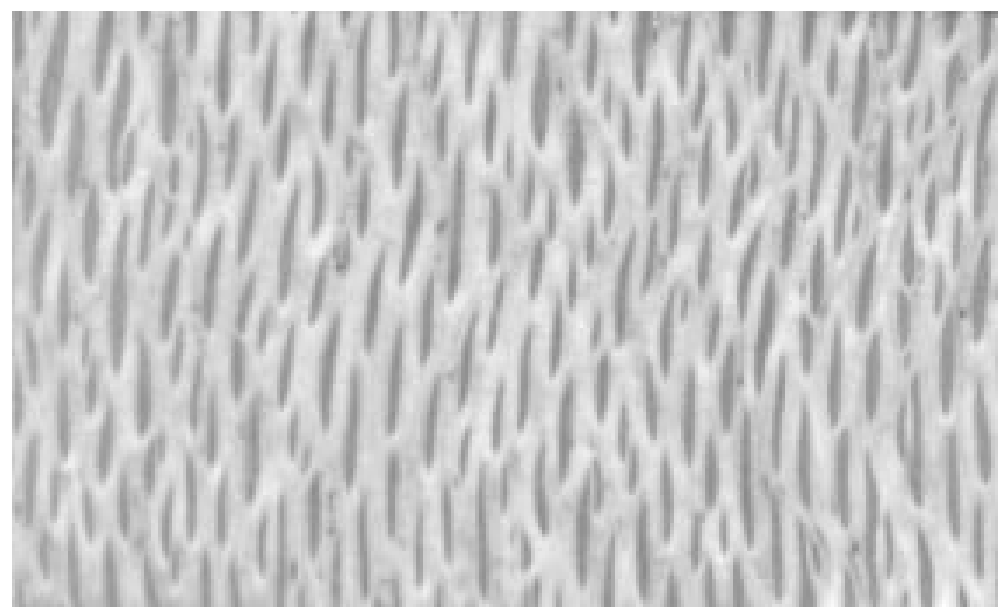

a.

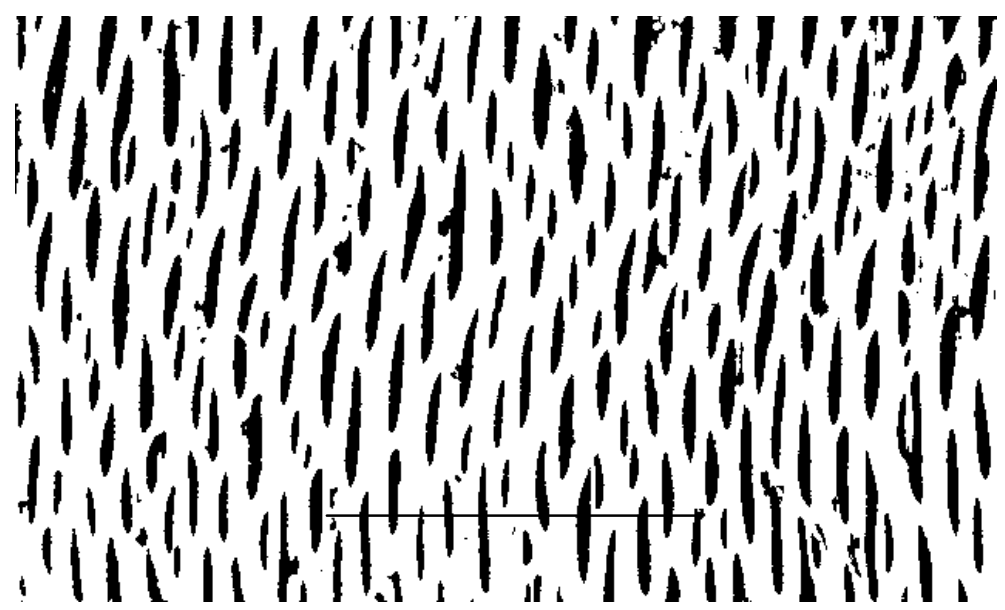

b. (la línea equivale a $1 \mathrm{~cm}$ )

Figura 4. Imágenes de una sección tangencial utilizada para estimar el porcentaje de área ocupada por radios.

debido al aplastamiento que ejerce la cuchilla de corte sobre las fibras y los radios. Aparentemente, las fibras tienen una menor densidad que los radios, lo que ocasiona que éstas se levanten posteriormente por encima de la línea de corte de la herramienta. En la figura 5 se presentan las fotografías de las superficies radial, tangencial y transversal de una pieza de grevilea de 5 por 5 por $15 \mathrm{~cm}$. Los resultados obtenidos permiten esperar que en operaciones de torneado, barrenado y escopleado, la superficie maquinada presente pocos defectos. 


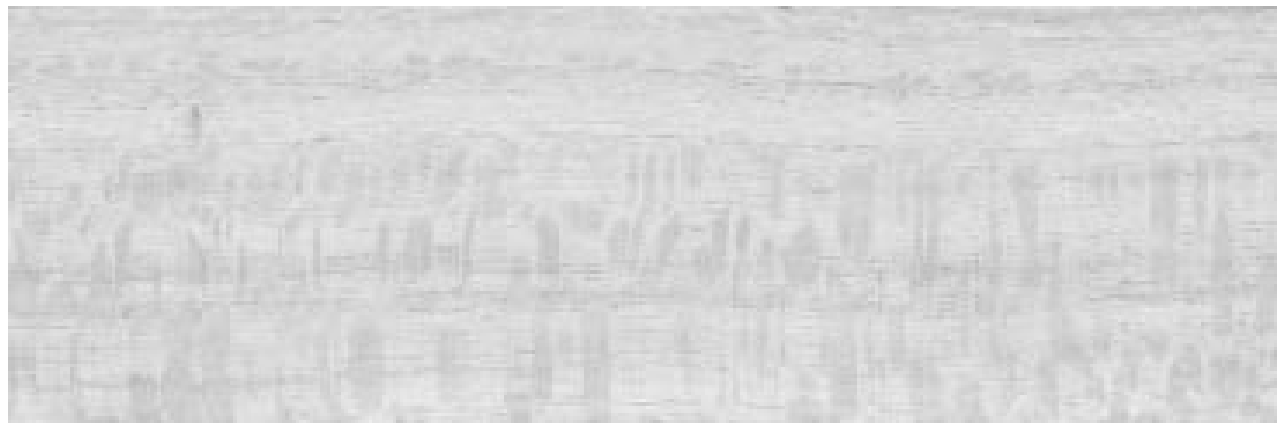

Cara radial

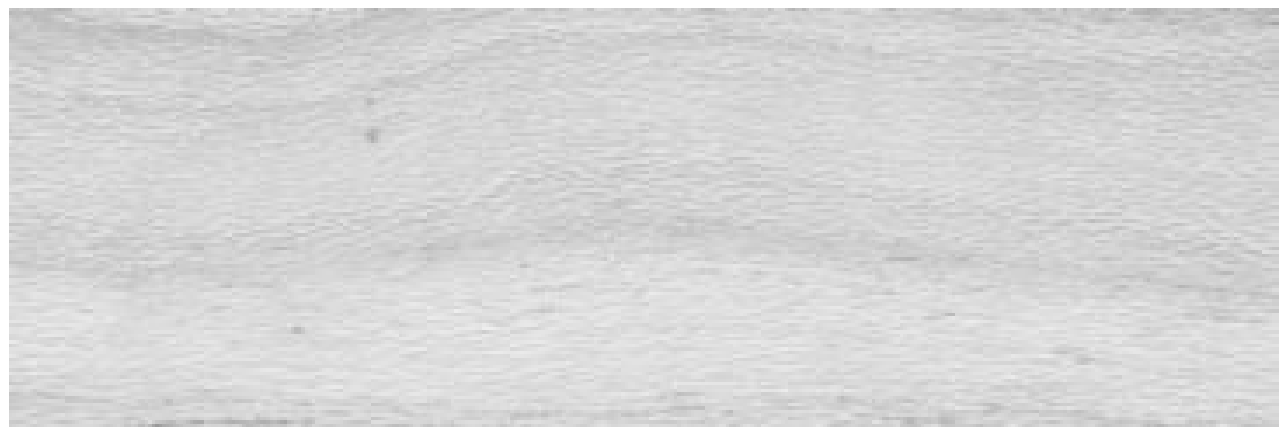

Cara tangencial

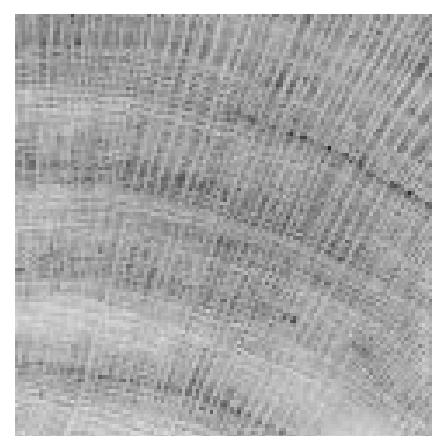

Cara transversal

Figura 5. Caras radial, tangencial y transversal de una pieza de grevilea de 5 por 5 por $15 \mathrm{~cm}$ 
Densidad relativa. La densidad relativa (PA/VV) media de la especie estudiada se obtuvo de un total de 273 especímenes, el valor medio obtenido fue 0.487 con $4 \%$ de coeficiente de variación.

Contracciones. Se obtuvieron los porcentajes total e intermedio de contracción radial y tangencial. Los resultados obtenidos son valor promedio $(\mathrm{m})$, número de probetas ensayadas $(\mathrm{n})$, desviación estándar (de) y coeficiente de variación (cv). Estos resultados se muestran en la Tabla 1. El coeficiente de anisotropía (contracción tangencial/radial) resulta de 2.69, lo que según Bárcenas (1995), se interpreta como una baja estabilidad dimensional.

De la pila de secado que se hizo para la elaboración de probetas en condición seca, se obtuvieron piezas con distorsiones leves, que permitieron la elaboración de las probetas sin mayor problema.

De la muestra de probetas de contracciones se obtuvo un PSF de $41.0 \%$.

Pruebas mecánicas. Los resultados obtenidos de las pruebas mecánicas se presentan en la Tabla 2 . Los resultados de pruebas realizadas en condición seca se ajustaron al $12 \%$ de $\mathrm{CH}$. El ajuste se hizo de acuerdo con Brown et al. (1952). No se encontró una diferencia significativa (menos de 1\%) entre los resultados obtenidos de dureza lateral en dirección radial y los obtenidos en dirección tangencial, por ello se presenta un resultado promedio de dureza lateral en general. En la Tabla 2 se presenta, además, una clasificación de algunas de las propiedades mecánicas de acuerdo con Dávalos y Bárcenas (1998 y 1999) y con Dávalos et al. (sin publicar), quienes hicieron una clasificación de algunas propiedades mecánicas de la madera con base en estudios experimentales en maderas mexicanas.

Se hicieron análisis de correlación entre las propiedades mecánicas y la densidad relativa, encontrando que, a pesar de la poca variación en la densidad relativa de la especie, no existe alguna relación entre ésta y las propiedades mecánicas. Sólo en los casos de dureza lateral y dureza en extremos se encontró una mayor correlación entre las variables, obteniendo 0.47 y 0.54 de coeficiente de determinación $\left(r^{2}\right)$, respectivamente. En el resto de las propiedades los coeficientes de determinación oscilaron alrededor de 0.1 .

Tabla 1. Resultados obtenidos de las pruebas de contracción radial y tangencial.

\begin{tabular}{|c|c|c|c|c|c|}
\hline & & $\begin{array}{c}\mathrm{CH} \\
\text { "verde" } \\
(\%)\end{array}$ & $\begin{array}{c}\mathrm{CH} \\
\text { INTERMEDIO } \\
(\%)\end{array}$ & $\begin{array}{c}\text { CONTRACCIÓN } \\
\text { INTERMEDIA } \\
(\%)\end{array}$ & $\begin{array}{c}\text { CONTRACCIÓN } \\
\text { TOTAL } \\
(\%)\end{array}$ \\
\hline radial & $\begin{array}{l}\mathrm{n} \\
\mathrm{m} \\
\mathrm{de} \\
\mathrm{cv}\end{array}$ & $\begin{array}{c}23 \\
133 \\
4.5 \\
3\end{array}$ & $\begin{array}{c}23 \\
8.3 \\
0.5 \\
6\end{array}$ & $\begin{array}{l}23 \\
2.0 \\
0.3 \\
13\end{array}$ & $\begin{array}{l}23 \\
2.6 \\
0.2 \\
10\end{array}$ \\
\hline tangencial & $\begin{array}{l}\mathrm{n} \\
\mathrm{m} \\
\mathrm{de} \\
\mathrm{cV}\end{array}$ & $\begin{array}{c}21 \\
133 \\
6.5 \\
5\end{array}$ & $\begin{array}{c}20 \\
8.4 \\
0.5 \\
6\end{array}$ & $\begin{array}{c}20 \\
6.1 \\
0.4 \\
6\end{array}$ & $\begin{array}{l}21 \\
7.5 \\
0.7 \\
9\end{array}$ \\
\hline
\end{tabular}


Tabla 2. Resultados de las pruebas mecánicas Condición seca.

\begin{tabular}{|c|c|c|c|c|c|c|}
\hline PRUEBA & PROPIEDAD & $\mathrm{n}$ & PROMEDIO & $\begin{array}{l}\text { DESVIACIÓN } \\
\text { ESTÁNDAR }\end{array}$ & CV (\%) & $\begin{array}{l}\text { CLASIFI- } \\
\text { CACIÓN }\end{array}$ \\
\hline $\begin{array}{l}\text { Densidad } \\
\text { relativa }\end{array}$ & $\mathrm{pa} / \mathrm{vv}$ & 273 & 0.487 & 0.021 & 4 & \\
\hline Flexión & $\begin{array}{l}\operatorname{ELP}\left(\mathrm{kg} / \mathrm{cm}^{2}\right) \\
\operatorname{MOR}\left(\mathrm{kg} / \mathrm{cm}^{2}\right) \\
\operatorname{MOE}\left(\mathrm{kg} / \mathrm{cm}^{2}\right)^{*} 1000 \\
\operatorname{TLP}\left(\mathrm{kg}-\mathrm{cm} / \mathrm{cm}^{3}\right)\end{array}$ & 12 & $\begin{array}{c}343 \\
682 \\
74 \\
0.091\end{array}$ & $\begin{array}{c}66 \\
60 \\
10 \\
0.031\end{array}$ & $\begin{array}{c}19 \\
9 \\
14 \\
35\end{array}$ & $\begin{array}{c}\mathrm{b} \\
\mathrm{mb}\end{array}$ \\
\hline $\begin{array}{l}\text { Compresión } \\
\text { paralela }\end{array}$ & $\begin{array}{l}\operatorname{ELP}\left(\mathrm{kg} / \mathrm{cm}^{2}\right) \\
\operatorname{MOE}\left(\mathrm{kg} / \mathrm{cm}^{2}\right)^{*} 1000 \\
E_{\text {máx. }}\left(\mathrm{kg} / \mathrm{cm}^{2}\right)\end{array}$ & 17 & $\begin{array}{c}128 \\
66 \\
267\end{array}$ & $\begin{array}{l}30 \\
12 \\
38 \\
\end{array}$ & $\begin{array}{l}24 \\
18 \\
14 \\
\end{array}$ & $\mathrm{mb}$ \\
\hline $\begin{array}{l}\text { Compresión } \\
\text { perpendicular }\end{array}$ & $\begin{array}{l}\operatorname{ELP}\left(\mathrm{kg} / \mathrm{cm}^{2}\right) \\
E_{1 \mathrm{~mm}}\left(\mathrm{~kg} / \mathrm{cm}^{2}\right)\end{array}$ & 15 & $\begin{array}{l}48 \\
79 \\
\end{array}$ & $\begin{array}{l}8 \\
7\end{array}$ & $\begin{array}{l}15 \\
11\end{array}$ & b \\
\hline Dureza & $\begin{array}{l}\text { Lateral }(\mathrm{kg}) \\
\text { Extremos }(\mathrm{kg})\end{array}$ & 15 & $\begin{array}{l}378 \\
358\end{array}$ & $\begin{array}{l}25 \\
23\end{array}$ & $\begin{array}{l}7 \\
6\end{array}$ & $\begin{array}{c}m \\
b\end{array}$ \\
\hline Cortante & $E_{\text {máx. }}\left(\mathrm{kg} / \mathrm{cm}^{2}\right)$ & 18 & 90 & 7 & 8 & b \\
\hline Tenacidad & $\mathrm{T}\left(\mathrm{kg}-\mathrm{cm} / \mathrm{cm}^{3}\right)$ & 43 & 2.132 & 0.440 & 21 & \\
\hline \multicolumn{7}{|c|}{ Condición "verde". } \\
\hline PRUEBA & PROPIEDAD & $\mathrm{n}$ & PROMEDIO & $\begin{array}{l}\text { DESVIACIÓN } \\
\text { ESTÁNDAR }\end{array}$ & CV (\%) & $\begin{array}{l}\text { CLASIFI- } \\
\text { CACIÓN }\end{array}$ \\
\hline Flexión & $\begin{array}{l}\operatorname{ELP}\left(\mathrm{kg} / \mathrm{cm}^{2}\right) \\
\operatorname{MOR}\left(\mathrm{kg} / \mathrm{cm}^{2}\right) \\
\operatorname{MOE}\left(\mathrm{kg} / \mathrm{cm}^{2}\right)^{*} 1000 \\
\operatorname{TLP}\left(\mathrm{kg}-\mathrm{cm} / \mathrm{cm}^{3}\right)\end{array}$ & 19 & $\begin{array}{c}273 \\
433 \\
51 \\
0.082\end{array}$ & $\begin{array}{c}31 \\
34 \\
58 \\
0.015\end{array}$ & $\begin{array}{c}11 \\
8 \\
11 \\
18\end{array}$ & $\begin{array}{c}\mathrm{b} \\
\mathrm{mb} \\
\mathrm{a}\end{array}$ \\
\hline $\begin{array}{l}\text { Compresión } \\
\text { paralela }\end{array}$ & $\begin{array}{l}\operatorname{ELP}\left(\mathrm{kg} / \mathrm{cm}^{2}\right) \\
\operatorname{MOE}\left(\mathrm{kg} / \mathrm{cm}^{2}\right)^{*} 1000 \\
E_{\text {máx. }}\left(\mathrm{kg} / \mathrm{cm}^{2}\right)\end{array}$ & 17 & $\begin{array}{c}118 \\
54 \\
177\end{array}$ & $\begin{array}{l}11 \\
14 \\
18\end{array}$ & $\begin{array}{c}9 \\
25 \\
10\end{array}$ & $\begin{array}{c}\mathrm{mb} \\
\mathrm{b}\end{array}$ \\
\hline $\begin{array}{l}\text { Compresión } \\
\text { perpendicular }\end{array}$ & $\begin{array}{l}\text { ELP }\left(\mathrm{kg} / \mathrm{cm}^{2}\right) \\
E_{1 \mathrm{~mm}}\left(\mathrm{~kg} / \mathrm{cm}^{2}\right)\end{array}$ & 16 & $\begin{array}{l}40 \\
80\end{array}$ & $\begin{array}{l}6 \\
9 \\
\end{array}$ & $\begin{array}{l}15 \\
11 \\
\end{array}$ & $\begin{array}{l}\mathrm{m} \\
\mathrm{m}\end{array}$ \\
\hline Dureza & $\begin{array}{l}\text { Lateral }(\mathrm{kg}) \\
\text { Extremos (kg) }\end{array}$ & 16 & $\begin{array}{l}343 \\
302 \\
\end{array}$ & $\begin{array}{l}19 \\
16 \\
\end{array}$ & $\begin{array}{l}6 \\
5\end{array}$ & $\begin{array}{l}\mathrm{m} \\
\mathrm{m}\end{array}$ \\
\hline Cortante & $E_{\text {máx. }}\left(\mathrm{kg} / \mathrm{cm}^{2}\right)$ & 15 & 69 & 4 & 6 & $\mathrm{~m}$ \\
\hline Tenacidad & $\mathrm{T}\left(\mathrm{kg}-\mathrm{cm} / \mathrm{cm}^{3}\right)$ & 45 & 3.160 & 0.686 & 22 & \\
\hline \multicolumn{3}{|c|}{ 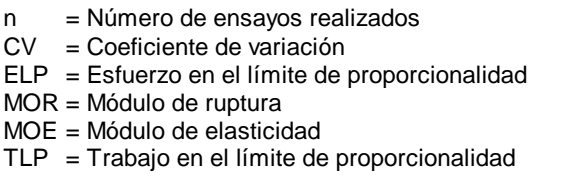 } & \multicolumn{2}{|l|}{$\begin{array}{ll}E_{\text {máx. }} & =\text { Esfuer } \\
\mathrm{T} & =\text { Energ } \\
\mathrm{Mb} & =\text { Muy b } \\
\mathrm{b} & =\text { Bajo } \\
\mathrm{m} & =\text { Medio } \\
\mathrm{a} & =\text { Alto }\end{array}$} & idad de volu & umen \\
\hline
\end{tabular}


Radios. En el cálculo del porcentaje del área de radios presentes en la madera, se encontraron valores hasta de un 35\% de radios en una sección tangencial, a pesar del número tan pequeño de secciones que se analizaron. El porcentaje promedio obtenido de área de radios es de 28.2 con $17 \%$ de coeficiente de variación. En promedio se encontraron 8.7 radios por centímetro con una muestra de 12 ejemplares y $8 \%$ de coeficiente de variación. En la figura 4 se ilustra la abundancia de radios que contiene esta madera.

\section{DISCUSIÓN}

De los resultados observados durante el maquinado de las probetas (cepillado y lijado), se puede esperar, en moldurado, barrenado y escopleado, una superficie con pocos defectos. Lo anterior, aunado a la agradable apariencia de las superficies resultantes del cepillado y lijado (producto, entre otras cosas, de la abundancia de radios) y a la escasa presencia de nudos grandes, hace que la madera de esta especie se pueda recomendar ampliamente para su uso en la fabricación de muebles.

Dada la baja variabilidad encontrada en la densidad relativa ( $p a / v v)$, se puede concluir que la madera de grevilea es muy homogénea en su densidad. De acuerdo con la clasificación de la estabilidad dimensional de la madera según sus contracciones, presentada por Bárcenas (1995), la madera de grevilea tiene un índice de susceptibilidad a las distorsiones y alabeos muy alto (Contracción tangencial $/$ radial $=2.69$ ), $\sin$ embargo, tomando en cuenta los buenos resultados obtenidos (distorsiones pequeñas) en el secado para elaboración de probetas en condición seca, se puede concluir que la inestabilidad dimensional de esta madera se puede atenuar con condiciones especiales de secado como las mencionadas en la metodología.

Se considera conveniente hacer un cálculo del PSF con un tamaño de muestra mayor. Se recomienda tomar el valor del PSF obtenido aquí sólo como un índice dado el tamaño de la muestra que se estudió.

Los resultados de propiedades mecánicas por Tanzania: Util. Div. For. Dep. (1962), que se presentan resumidos en Chudnoff (1984), así como los presentados en la página de internet "ForestWorld" (2000) son similares a los obtenidos en este trabajo. Por ejemplo, Tanzania: Util. Div. For. Dep. (1962) menciona un valor de $596 \mathrm{~kg} / \mathrm{cm}^{2}$ de módulo de ruptura en flexión, mientras que ForestWorld indica $768 \mathrm{~kg} / \mathrm{cm}^{2}$, lo que significa un $13 \%$ abajo y un $13 \%$ arriba del resultado obtenido en esta investigación, respectivamente. Otro ejemplo puede ser la resistencia en dureza lateral, para la que Tanzania: Util. Div. For. Dep. (1962) señala 382 kg y ForestWorld $381 \mathrm{~kg}$, lo que difiere del resultado obtenido en este trabajo un $1 \%$.

Como se aprecia en la Tabla 2, Grevillea robusta se puede clasificar como una especie de baja resistencia mecánica, excepto en su trabajo en el límite de proporcionalidad en flexión, dureza y compresión perpendicular. Incluso, comparando los resultados obtenidos con la resistencia mecánica de los pinos de densidad semejante (Ordóñez et al., 1989, Echenique y Díaz, 1972), se encuentra que la grevilea tiene valores menores de resistencia. Tomando en cuenta que la resistencia en compresión perpendicular y en dureza de esta especie no es baja, se puede recomendar esta madera para su uso en durmientes y en pisos. Por otro lado, dada su baja resistencia en las demás propiedades y las pequeñas longitudes de fuste comercial de la especie, sólo se puede recomendar para su uso en la 
construcción de estructuras ligeras, utilizando piezas cortas.

Los coeficientes de variación obtenidos para todas las propiedades mecánicas y la densidad están por debajo de los presentados por Bárcenas y Fuentes (1999) para especies mexicanas, los cuales varían entre14 y $27 \%$.

\section{CONCLUSIONES}

- Por la agradable textura, color, brillo y lustre que se logra al maquinar la madera de esta especie, y su trabajabilidad, se puede recomendar ampliamente para su uso en muebles y torneado, lo que le daría un amplio valor agregado después de su uso como árbol de ornato o de sombra.

- La madera de grevilea es muy homogénea en su densidad relativa.

- Sólo se puede utilizar para estructuras ligeras con piezas cortas, dados su baja resistencia y el tamaño de fuste comercial de los árboles.

- El secado de esta madera se debe realizar con secuelas suaves, ejerciendo presión constante sobre la pila de secado para evitar distorsiones grandes.

- Es necesario hacer una investigación más a fondo sobre esta especie, dadas sus características anatómicas y con ella determinar si existe una relación entre éstas y sus propiedades mecánicas.

\section{RECONOCIMIENTOS}

Este trabajo se realizó con el apoyo de los recursos fiscales asignados al Departamento de Productos Forestales y
Conservación de Bosques del Instituto de Ecología, A.C. con la clave 902-13.

\section{REFERENCIAS}

ASTM. 1997. Standard methods for testing small clear specimens of timber. ASTM D143-94. Phyladelphia, PA. Ameriacan Society for Testing and Materials:23-53.

Bárcenas P., G. M. 1995. Caracterización tecnológica de 20 especies maderables de la Selva Lacandona, Chis., México. Madera y Bosques $1(1): 9-38$.

Bárcenas P., G. M. y M. Fuentes S. 1999. Propiedades físicas y mecánicas de la madera. In: Manual de construcción de estructuras ligeras de madera. Comisión Forestal de América del Norte. 2a․ ed. p. 27. 476 p.

Brown, H. P., A. J. Panshin y C. C. Forsaith. 1952. Textbook of Wood Technology. Vol. II. Ed. McGraw-Hill. EUA.

Choong, E. T. y S. S. Achmadi. 1991. Effect of extractives on moisture sorption and shrinkage in tropical woods. Wood and Fiber Science. Vol. 23. No. 2:185-196.

Chudnoff M. 1984. Tropical timbers of the world. United States Department of Agriculture. Forest Service. Agriculture Handbook Number 607. Madison, Wis. EUA. 464 p.

Dávalos S., R. y G. Bárcenas P. 1998. Clasificación de las propiedades mecánicas de las maderas mexicanas en condición "verde". Madera y Bosques 4(1):65-70.

Dávalos S., R. y G. Bárcenas P. 1999. Clasificación de las propiedades mecánicas de las maderas 
mexicanas en condición "seca". Madera y Bosques 5(1):61-69.

Dávalos S., R., R. P. Zárate M. y C. De la Paz P. O., Tablas de clasificación de tres propiedades mecánicas de maderas mexicanas en condición "verde". Madera y Bosques 7(1):7177.

Echenique M., R. y V. Díaz G. 1972. Algunas características tecnológicas de la madera de once especies mexicanas. SAG. SFF. INIF. México. $71 \mathrm{p}$.

ForestWorld. 2000. Woods of the world. www.forestworld.com

INECOL. 2000. Manual de procedimientos de pruebas en pequeñas probetas de madera libre de defectos. Instituto de Ecología, A. C. Xalapa, Ver. 91 p.

Longwood, F. 1961. Puerto Rican woods. Their machining, seasoning and related characteristics. Agriculture handbook. No. 205. Department of Agriculture. Forest Service. 34 p.

Nee, M. 1988. Proteaceae. Flora de Veracruz. Fasc. 56. INIREB. Xalapa, Ver. $8 \mathrm{p}$.

Niembro R., A. 1986. Árboles y arbustos útiles de México. Ed. LIMUSA. México. 206 p.

Ordóñez C., V. R., G. Bárcenas P. y A. Quiroz S. 1989. Características físico-mecánicas de la madera de diez especies de San Pablo Macuiltianguis Oaxaca. La madera y su uso. No. 21. Xalapa, Ver.»

1 Departamento de Productos Forestales y Conservación de Bosques. Instituto de Ecología, A. C. Apdo. Postal 63. Xalapa, Ver. 91000 México. c.e. zaratep@ecologia.edu.mx

Manuscrito recibido el 1 de noviembre de 2000

Aceptado el 2 de febrero de 2001

Este documento se debe citar como:

Zárate M., R. P., V. R. Ordóñez C. y J. L. Martínez C. 2001. Determinación de algunas propiedades físicas y mecánicas de Grevillea robusta A. Cunn. del estado de Veracruz. Madera y Bosques 7(1):57-69. 